Revista de Estudios Histórico-Jurídicos

[Sección Derecho Romano] XXXI (Valparaíso, Chile, 2009)

[pp. 159 - 168]

\title{
LA FÓRMULA DE LA "AGNITIO BONORUM POSSESSIONIS" EN EL SIGLO III*
}

[““'Agnitio Bonorum Possessionis” Formula in the Third Century”]

\author{
Diego M. Santos**
}

\section{Resumen}

El trabajo examina la fórmula de la agnitio bonorum possessionis posiblemente usada al menos en el siglo III, a la luz de los papiros conservados, en que se documenta tal trámite sucesorio y una referencia a él en la Paraphrasis de Theophilus antecessor.

Palabras Clave: Agnitio bonorum possessionis - Fórmula de la agnitio - Papiros con casos de agnitio.
Abstract

This work examines the agnitio bonorum possessionis formula, possibly used at least in the $3^{\text {rd }}$ century, in the light of the conserved papyrus, in which this succession procedure is documented and it is referred to in Theophilus antecessor's Paraphrasis.

KeYwORDS: Agnitio bonorum possessionis - Agnitio's formula - Papyrus with cases of agnitio.

* Una versión preliminar de este trabajo fue leída en las VII Jornadas de Estudios Medievales, organizadas por el Departamento de Estudios Medievales del Instituto Multidisciplinario de Historia y Ciencias Humanas del Consejo Nacional de Investigaciones Científicas y Técnicas y la Sociedad Argentina de Estudios Medievales, el día 4 de septiembre de 2007.

** Miembro del Centro Argentino de Estudios Bizantinos. Dirección postal: Humaitá 1764, C.P. 1700, Tapiales, Provincia de Buenos Aires, Argentina. Correo electrónico: diegoaug@ yahoo.com.ar 


\section{INTRODUCCIÓN}

La pequeña cantidad de documentos que contienen una agnitio bonorum possessionis, o petición de la sucesión pretoria (bonorum possessio, petición que Marcelo, en D. 38,15,5 pr., llama precisamente "agnitio"), descubiertos en Egipto fue comentada desde principios del siglo XX, especialmente en relación con la existencia del edicto provincial ${ }^{1}$ y los alcances de la Constitutio Antoniniana ${ }^{2}$. Intentaremos llamar la atención sobre la estructura de la fórmula procesal de los mismos, que permaneció sin ser directamente señalada hasta la actualidad ${ }^{3}$. La referencia a ésta podrá resultar de utilidad en la reconstrucción o identificación de ejemplares descubiertos o por descubrirse. También se pondrá atención en la exactitud de la mención a la fórmula de Teófilo antecessor, comentarista bizantino de las Institutiones de Justiniano,.

El hecho de haber habido una fórmula para la agnitio bonorum possessionis

${ }^{1}$ La primera mención parece ser EgER, O., Agnitio bonorum possessionis vom Jahre 249 p. C., en Zeitschrift der Savigny-Stiftung rom. Abt. 32 (1911), pp. 378-382; véase, especialmente, la p. 382. Como señala KaTzOFf, R., The Provincial Edict in Egypt, en Tijdschrift voor Rechtsgeschiedenis/ Revue d'Histoire du Droit 37 (1969) 3-4, p. 417: "Eger was followed in this interpretation by all the writers on the subject".

${ }^{2}$ Sobre ella, véanse: Bell, H. I., P. Giss. 40 and the "Constitutio Antoniniana", en Journal of Egyptian Archaeology 28 (1942), pp. 39-40; Bell, H. I., The "Constitutio Antoniniana" and the Egyptian Poll-Tax, en Journal of Roman Studies 37 (1947) 1-2, pp. 17-23; D’ORs, A., Estudios sobre la "Constitutio Antoniniana”, en Emerita 11 (1943), pp. 332-337; trabajo éste continuado en: Anuario de Historia del Derecho Español 15 (1944), pp. 162-204; Anuario de Historia del Derecho Español 17 (1946), pp. 586-604; Sefarad 6 (1946), pp. 21-36; Emerita 24 (1956), pp. 1-26; D'Ors, A. - Gundel, H. G., Zur Constitutio Antoniniana (P. Giss. 40 I). Kurzberichte aus den Giessaner Papyrus-Sammlung Nro. 22 (Giessen, 1973); HeICHELHEIM, F. M., The Text of the Constitutio Antoniniana and the Three Other Decrees of the Emperor Caracalla contained in Papyrus Gissensis 40, en Journal of Egyptian Archaeology 26 (1941), pp. 10-22; Jones, A. H. M., Another Interpretation of the "Constitutio Antoniniana", en Journal of Roman Studies 26 (1936) 2, pp. 223-235; Keresztes, P., The "Constitutio Antoniniana" and the Persecutions of Caracalla, en The American Journal of Philology 91 (1970) 4, pp. 446-459; LAISTNER, M. L. W., "Dediticii”: the Source of Isidore (Etym. 9.4.49-50), en Journal of Roman Studies 11 (1921), pp. 267-268; MEYER, P. M., Griechische Papyri im Museum des oberhessischen Geschichtvereins zu Gissen (P. Giss.) (Leipzig - Berlin, Teubner, 1910); Meyer, P. M., Juristische Papyri, Erklärung von Urkunden zur Einführung in die Juristische Papyruskunde (Berlin, Weidmannsche Buchhandlung, 1920); MitTeIs, L. - WiLCKEN, U., Grundzüge und Chrestomathie der Papyruskunde (Hildesheim, Georg Olms Verlagsbuchhandlung, 1963), II, 2; Oliver, J. H., Free Men and "Dediticii, en The American Journal of Philology 73, (1955)

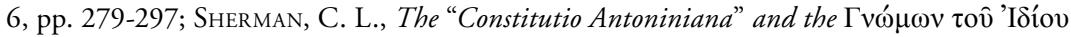

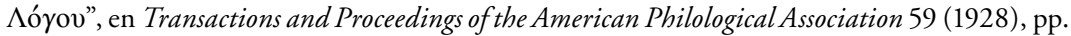
33-47; Sherwin-White, A. N., The Tabula of Banasa and the "Constitutio Antoniniana", en Journal of Roman Studies 63 (1973), pp. 86-98; Wilhelm, A., Die "Constitutio Antoniniana”, en American Journal of Archaeology 38 (1934) 1, pp. 178-181. Véase también la bibliografía citada en Bancalari Molina, A., Relación entre la "Constitutio Antoniniana" y la "Imitatio Alexandri” de Caracalla, en Revista de Estudios Histórico-Jurídicos 22 (2000), pp. 17-30.

${ }^{3}$ Solo EgER, O., cit. (n. 1), p. 380, observó la similitud entre el documento que publica (P. Giessen Inv. Nro. 40) y la Paraphrasis a las Institutiones de Teófilo, sin profundizar en la misma. 
es conocido a través de una constitución dada por Constancio II (337-361) en Laodicea, en febrero de 339, y que se conserva en CI. 6,9,9. Al comienzo del texto se alude, en efecto, a una medida adoptada anteriormente, en orden a abolir el empleo de palabras (solemnes), que van calificadas de "engaños de palabras vacías", en función de lo que ahí se denomina "amplecteri hereditatis": "ut verborum inanium excludimos captiones". Esta aceptación sucesoria verbis, mencionada por Constancio, es recordada en una interpretatio visigótica del "Breviario de Alarico", a la constitución de Teodosio II y Valentiniano III recogida en CTh. 4,1,1; que reza así: "Interpretatio": Cretio et bonorum possessio antiquo iure a pretoribus petebatur: quod explanari opus non est, quia legibus utrumque sublatum est. Et ideo infans, licet loqui non possit, tamen hereditatem sibi debitam capit, cui morenti pater aut si, qui proximus fuerit, ex lege succedit". Como se ve, el texto alude a la petición hecha a los pretores según el antiquum ius, y advierte que ella (junto a la cretio) fue abolida por las leyes ("legibus utrumque sublatum est").

¿Cuál era la fórmula referida por Constancio? La única mención se encuentra en la Paraphrasis a las Institutiones del ya mencionado Teófilo antecessor (siglo $\mathrm{VI})^{4}$, donde se complementa Inst. 3,9,125 con una valiosa referencia a la fórmula anteriormente utilizada: "es necesario ir al pretor y decir especialmente: 'dame

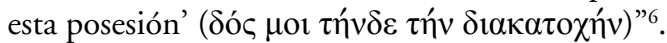

Se han conservado varios ejemplares de agnitio bonorum possessionis ${ }^{7}$, todos datados en el siglo III. La importancia de la Constitutio Antoniniana se refleja no solo en la expansión de esta institución del Derecho pretoriano ${ }^{8}$, sino también en la onomástica de los documentos, pues casi todas las personas mencionadas llevan

${ }^{4}$ Institutionum Graeca paraphrasis. Theophili Antecessori vulgo tributa, ad fidem librorum manu scriptorum recensuit, prolegominis, notis criticis, versione Latina instruxit E. C. Ferrini. Accedit espistula C. E. Zacharia von Ligenthal (reimpresión Aalen, Scientia, 1967), dos volúmenes.

${ }^{5}$ Inst. 3,9,12: "Sed bene anteriores principes et huic causae providerunt, ne quis pro petenda bonorum possessionem curet, sed, quocumque modo si addmittentis eam iudicium intra statuta tamen tempora ostenderit, plenum habeat earum beneficium".

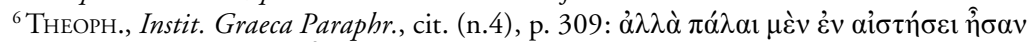

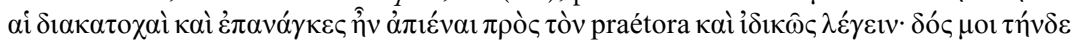

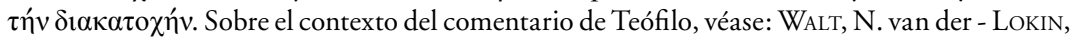
J. H. A., Historiae iuris Graeco-Romani Delineatio: les sources du Droit byzantin de 300 à 1453 (Groningen, 1985), pp. 38-46; EGER, cit. (n.1), p. 380, sigue a GLÜCK, C. F. von, Ausführliche Erläuterung der Pandecten (Erlangen, Balm \& Enfe, 1870), XXXVI-XXXVIII, erster Theil, p. 89, cuya traducción del texto griego no es, sin embargo, muy apropiada, pues el acusativo $\tau \eta ́ v \delta \varepsilon$ debe ser traducido como "hanc", no como "illam".

${ }^{7}$ La lista más reciente se encuentra en Gagos, T. - Heilporn, P., A New "agnitio bonorum possessionis", en Gagos, T. - Bagnall, R. S., Essays and Texts in Honor of J. David Thomas (Oakville, The American Society of Papyrologists, 2001), pp. 175-185, especialmente pág. 176-178. A este grupo hay que añadir un texto inédito, el P. Duke 641 (P. Miss. 32), conservado en la Duke University, Durham, North Carolina.

${ }^{8} \mathrm{La}$ opinión generalizada se inclinaba por la poca frecuencia de estos documentos, explicándola por la poca frecuencia de empleo de esta institución en Egipto. Cfr. KaTzoff, R., cit. (n. 1), p. 418, quien sigue a KRELLER, H., Erbrechtliche Untersuchungen auf Grund der graeco-aegyptishcen Papyruskunden (Leipzig - Berlin, 1919), pp. 125-162. Katzoff expresa que "subsequent discoveries seem to confirm this conclusion". Al contrario, Gagos y Heilporn 
el nomen Aurelius, tomado evidentemente de aquel del emperador Caracalla, cuya concesión de ciudadanía llegó a los metropolitanos egipcios. Estos ejemplares se vuelven la fuente principal para describir la fórmula utilizada.

En cuanto a sus orígenes, es necesario mencionar que todos los documentos proceden de Oxirrinco. Ésto, más que deberse a algún tipo de particularidad, puede encontrar su explicación en lo azaroso de la conformación del registro arqueológico.

La inexistencia de documentos semejantes para la época anterior al siglo III puede deberse a la escasa aplicación del Derecho romano en Egipto (entre los pocos ciudadanos residentes) antes de la Constitutio Antoniniana, y la dificultad de encontrar papiros latinos en ciudades con una posible mayor población de ciudadanos romanos como Alejandría (donde las condiciones ecológicas no permiten la conservación de este tipo de material). Los documentos dejan de aparecer a fines del siglo III. Puede deberse a la pronta desaparición del uso de la fórmula en la primera mitad del siglo IV.

\section{ANÁLISIS DE LOS DOCUMENTOS}

La petición comienza con el nombre del magistrado a quien va dirigida (en

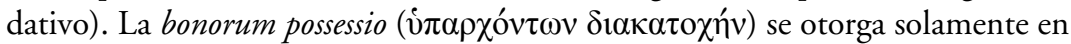
un tribunal ${ }^{10}$. El prefecto de Egipto es el máximo tribunal de esa provincia, siendo también la instancia a la que acuden los ciudadanos romanos que en esta residen,

sostienen, con mucho criterio, que: "given a short time-span, our evidence is not so small, at least for the Oxyrhynchite nome".

${ }^{9}$ Los papiros utilizados son: i) P. Ryl. IV 610 (223 d. C., en Roberts, C. H. - Turner, E. G., Catalogue of the Greek and Latin Papyri in the John Rylands Library, Manchester (Manchester, 1952, IV. Aún no hemos podido revisar un fragmento del mismo papiro, publicado por Daris, en Janeras, S. (editor), Miscelània Papirògica Ramon Roca-Puig en el seu vuitantè aniversari (Barcelona, Fundació Salvador Vives Casajuana, 1987). ii) P. Oxy. XLIII 3108 (ca. 240 d. C.). Véase sobre él: ReA, J. R., The Oxyrhynchus Papyri, Part XLIII (London, Egypt Exploration Society, 1975). Su imagen digitalizada puede encontrarse en: http://www.papyrology.ox.ac.uk/POxy/; iii) P. Giss. Bibl. Un., Inv. Nro. 40 (249 d.C.), en Meyer, P. M., Juritische Papyri, Erklärung von Urkunden zur Einführung in die Juristische Papyruskunde, (Berlin, Weidmannsche Buchhandlung, 1920), p. 73-75; sobre el cual D'Ors, A., Introducción al Estudio de los Documentos del Egipto Romano (Madrid, Instituto Antonio de Nebrija, 1948), pp. 190, 204-205. La imagen digitalizada de este manuscrito, al igual que del siguiente (SB IV 9298) puede ser encontrada en http://digibib.ub.uni-giessen.de/cgi-bin/populo/pap.pl); iv) P. Oxy. IX 1201 (258 d. C.), en Hunt, A. S., The Oxyrhynchus Papyri, Part IX (London, Egypt Exploration Fund, 1912); v) P. Mich. 1946 (269 d.C.), en Gagos, T. - Heilporn, P., A new "agnitio bonorum possessionis", en Gagos, T. - Bagnall, R. S., Essays and Texts in Honor of J. David Thomas (Oakville, The American Society of Papyrologists, 2001), pp. 175-185, plate 16 [Agradecemos a Traianos Gagos, de la Universidad de Michigan, por habernos facilitado la transcripción y edición de este papiro]. La imagen digitalizada del papiro puede ser consultada en el sitio web APIS: http://www.columbia.edu/cu/lweb/projects/digital/ apis/index.html. También se utilizó el P. Duke 641 = P. Miss. 32 (mediados del s. III d. C.), la imagen digitalizada del cual puede consultarse en: http://odyssey.lib.duke.edu/papyrus/ records/466.html .

${ }^{10}$ UlPIANO, ad Edictum, lib. XXXIX (D. 37,3,8): "Si causa cognita bonorum possessio detur, 
por lo que se trata siempre de este magistrado. La petición debe ser dirigida en latín y lleva una traducción griega, preparada para el archivo local. El uso de las dos lenguas se entiende: debido a que la petición y el otorgamiento, según el Derecho romano, debían ser realizados en latín; mientras que el resumen, la ubicación en el archivo, y sobre todo la traducción, se realizaban en griego, la lengua de la administración en el Egipto romano. Es sumamente probable que el peticionante, aunque ahora ciudadano, desconociese la lengua latina ${ }^{11}$.

La traducción griega puede variar y es evidente, como se observa a través de las diferencias presentadas por los documentos, que era realizada en cada caso particular, sin contar con una versión estereotipada para la traducción ${ }^{12}$.

Continúa el nombre, filiación y origen de quien dirige la petición, siguiendo a la preposición " $a b$ ”. En los documentos encontramos personas sui iuris o alieni iuris

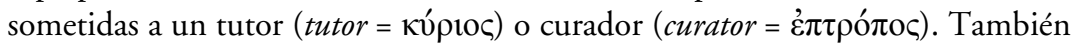
encontramos a un impúber con su padre como tutor ["impubere t(utore) $a$ (uctore)

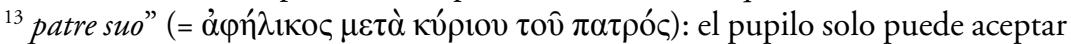
la bonorum possessio a través de su tutor ${ }^{14}$, aunque en una epístola dada posteriormente, por los emperadores de la segunda Tetrarquía (305-307), se menciona que si un juez da a un impúber la posesión de los bienes, sin la mediación de su tutor, se considera de todas formas un derecho adquirido ${ }^{15}$. En el P. Mich. inv. 1946, col. II, lin. 4, quien dirige la petición lo hace también en nombre de sus

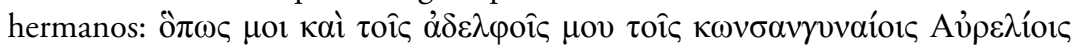

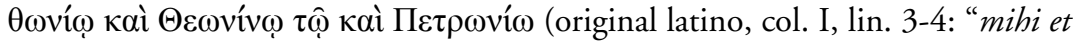
fratibus meis [consanguineis Aureliis Thonio et Theo]nino q(ui) e(t) Petronae]".

Luego de esto, se introduce la frase "rogo, domine, des mihi bonorum posses-

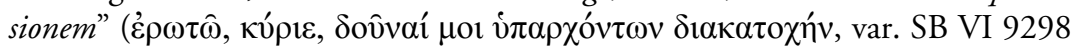

non alibi dabitur, quam pro tribunali, quia neque decretum de plano interponi, neque causa cognita bonorum possessio alibi, quaru pro tribunali dare potest".

${ }^{11}$ Un interesante comentario sobre el uso del latín y las traducciones griegas en este tipo de documentos legales se encuentra en AdAms, J. N., "Romanitas" and the Latin Language, en The Classical Quarterly 53.1 (2003), pp. 184-205, especialmente pp. 187-188. Véase también: Adams, J. N., Bilingualism and the Latin Language (Cambridge, Cambridge University Press, 2004), pp. 527-641, especialmente pp. 564-571

${ }^{12}$ El proceso para la agnitio bonorum possessionem, que no discutiremos aquí, puede encontrarse comentado en Ch.L.A. XI 486, pág. 28, cf. Gagos, T. - Heilporn, P., A New “agnitio bonorum possessionis", cit. (n. 7), pp. 175-176.

${ }^{13}$ La abreviatura "t.a." ("tutore auctore") se encuentra registrada en VAlERIO ProBo, De iuris notarum, V,17, en BaVIERA, J. - Furlani, J., Fontes Iuris Romani Anteiustiniani (Florentiae, apud S. A. G. Barbèra, 1940), II, p. 457.

${ }^{14}$ Justiniano, Institutiones 1,21,1: "Neque tamen hereditate adire, neque bonorum possessionem petere, neque hereditatem ex fideicomisso suscipere aliter possunt, nisi tutoris auctoritate, quamvis illis lucrosa sit, neque nullum damnum habeant".

${ }^{15}$ CI. 6,9,7: "Pars epistulae Constantii et Maximinai AA. et Severi et Maximini nobilissimorum CC. [a. 305]: Bonorum quidem possessionem pupilli nomine agnoscere tutorem potuisse, aperte declaratur. 1. Ipse autem pupillus bonorum possessionem sine tutoris auctoritate amplecti non potest, nisi etiam impuberi sine tutoris auctoritate hoc postulanti, sciens hoc, competens iudex dedit bonorum possessionem; tunc enim emolumentum sucessionis videtur praetorio iure quaesitum esse". 


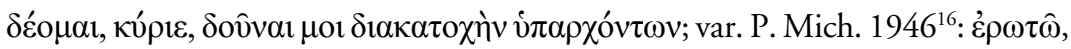

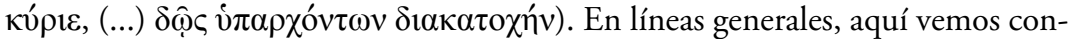

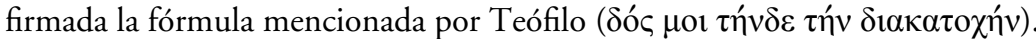

La primera parte (“rogo, domine”) es, quizás, una introducción respetuosa, no particular a la petición de bonorum possessio, probablemente utilizada durante el siglo III. Esto puede ser demostrado con los P. Oxy. $720^{17}$ (año 247), P. Oxy. 1466 (año 245) y P. Mich. 165 (año 236) ${ }^{18}$. Estos tres documentos son peticiones de tutores otorgados según la lex Iulia et Titia ${ }^{19}$ y todos proceden de Oxirrinco.

Utilizan la misma introducción: "rogo, domine, des mihi auctorem e lege Iulia (et) Titia" [reconstruido; var. "Iulia Titia" en P. Oxy. IV, 720] et ex senatus consulto [...]" (esto último se encuentra reconstruido por los editores de P. Mich 165; =

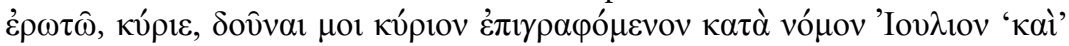

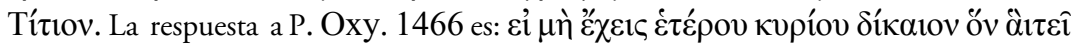

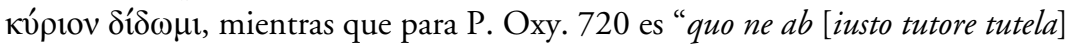
abeat Pl[utammonem] e leg(e) Iul(ia) et [Titia auctorem] do" y (probablemente en otra mano) "cepi"20.

Esto permite aislar las fórmulas utilizadas en ambos casos: "des mihi bonorum possessionem" y "des mihi auctorem". Quizá esta sea la parte más importante de

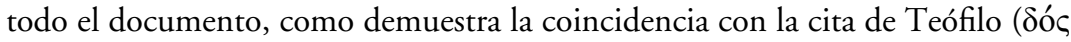

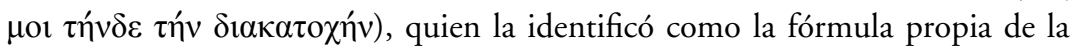
agnitio bonorum possessionis.

La separación entre "rogo, domine" y "des mihi bonorum possessionem" también es evidente en P. Mich. 1946, donde ambas se encuentran separadas por la identificación de los medio hermanos de quien redacta el documento: "rogo, domine, mihi et fratibus meis consanguineis Aureliis Thonio et Theonino qui et Petronae ex eode $(m)$ patre qui supra, matre Hieraciaine, des b(onorum) p(ossessionem)."

Una vez enunciada, a continuación de la fórmula, la persona en cuyos bienes se sucede y la relación del peticionario con ésta, se continúa así: "ex ea parte edicti qua

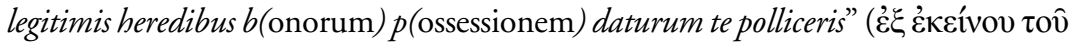

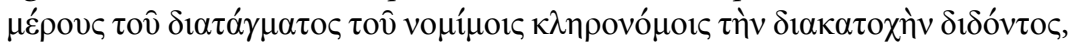

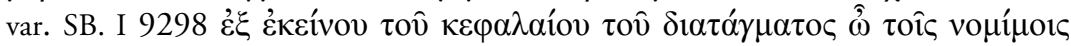

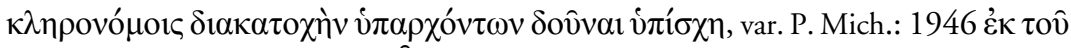

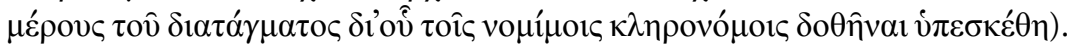
La abreviatura " $b$. p.", cuyo obvio sentido lo otorga el contexto, no se encuentra registrada por Valerio Probo. Es interesante notar que, a excepción de P. Oxy.

${ }^{16}$ Se debe siempre tener en cuenta las particularidades de este documento.

${ }^{17}$ Fotografía: Grenfell, B. P. - Hunt, A. S., The Oxyrrhynchus Papyri, Part IV (London, Egypt Exploration Society, 1904), plate VII; Thompson, E. M., An Introdution to Greek and Latin Paleography, (Oxford, Clarendon Press, 1912), pp. 320-324, imagen parcial en p. 325 (descrita erróneamente como "Facsimile No. 109", pues, en realidad, corresponde al No. 108).

${ }^{18}$ Inv. 3805; Winter, J. G. y otros, Michigan Papyri, III, (Ann Arbor, University of Michigan, Studies, Humanistic Series 40, 1936), Plate IIb.

${ }^{19}$ Inst. 1, 20. Cf. “Introducción” a P. Oxy. 1466 (p. 193).

${ }^{20}$ Véase la nota 10 , p. 195 , de P. Oxy. 1466, en que se menciona el P. Tebt. 397, donde se observa la misma institución en la ley greco-egipcia. 
IX 1201 y P. Mich. 1946, mientras que en la fórmula de petición se introduce la expresión completa “bonorum possessionem”, aquí se utiliza la abreviatura " $b$.p. p. Es probable que se deba a que en el primer caso se trata de la fórmula propiamente dicha y se requiera su escritura completa para la validez del documento.

Este es el sustento legal necesario para realizar la petición. En P. Oxy. 720, P. Oxy. 1466 y P. Mich. 165, el sustento invocado para la designación del tutor es precisamente la ley Iulia (et) Titia. Aquí es un edicto. Sin introducirnos en la larga discusión sobre a qué edicto hace referencia, podemos afirmar rápidamente que siendo una petición latina según el Derecho romano, debe tratarse necesariamente del edicto del pretor urbano. La atribución al edicto provincial la creemos por lo menos descontextualizada ${ }^{21}$.

Es probable que se trate más precisamente de la "pars tertia”, $\$ 142-165$, del Edictum perpetuum, donde aparentemente el pretor regulaba la bonorum possessio ${ }^{22}$. El uso de la variante $\kappa \varepsilon \varphi \alpha ́ \lambda \alpha 10 \varsigma=$ "caput" ${ }^{23}$ refuerza esta interpretación. "Daturum te polliceris" es parte de la fórmula, pero alude a la promesa edictal de la bonorum possessio unde legitimi.

Es interesante citar aquí la única valoración de esta fórmula, realizada por R. Katzoff: "the possibility still suggests itself that the notaries who drew up the documents only knew the formula for agnitio bonorum possessionis unde legitimi, and applied it regardless of its technical appropriatness. Such inexpert use of notarial formulas is not unknown in Egypt"24. Si bien Katzoff solo conocía tres de los documentos, es probable que la fórmula de la petición unde legitimi haya cumplido varias funciones en Egipto.

A continuación se coloca la fecha latina de la petición. El texto latino es seguido por un resumen griego. Al pie del resumen se coloca, en griego, la fecha en la cual se otorga la posesión de los bienes y, en latín, la suscripción (en otra mano) "ex edicto, recognovi (var. "legi"), por el cual el prefecto la otorga. Se registra a conti-

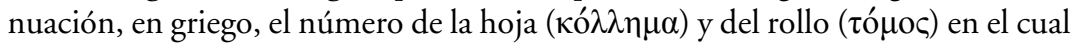
se archiva la petición y el otorgamiento de la misma. El documento termina con la traducción al griego del texto latino ( $\dot{\varepsilon} \rho \mu \varepsilon v \varepsilon i ́ \alpha$ var. $\left.\dot{\varepsilon} \rho \mu \varepsilon v \varepsilon i ́ \alpha \tau \hat{\omega} v{ }^{\circledR} \mathrm{P} \omega \mu \alpha \ddot{\kappa} \hat{\omega} v\right)$. Como recuerda Ulpiano ${ }^{25}$, los ascendientes y descendientes tienen el plazo de un año para pedir la bonorum possessio, mientras que todos los demás tienen cien días. Esto último es comentado en una constitución de Severo y Caracalla (205

${ }^{21} \mathrm{Y}$, así, coincidimos con Katzoff, R., cit. (n. 1), pp. 415-437.

${ }^{22}$ Lenel, O., Das Edictum Perpetuum, ein Versuch zu seiner Wiederherstellung (3a edición, Leipzig, Bernhard Tauchnitz, 1927), pp. 43, 180-183, 342-361. También puede encontrarse, aunque sin los comentarios de Lenel, en Riccobono, S., Fontes Iuris Romani Anteiustiniani, Pars Prima: Leges (Florentiae, apud S. A. G. Barbèra, 1941), pp. 335-391.

${ }^{23}$ Liddell, H. G. - ScotT, R., A Greek-English Lexicon (19 a edición, Oxford, Clarendon Press, 1996), p. 945a.

${ }^{24}$ Katzoff, R., cit. (n. 1), p. 421.

${ }^{25}$ UlPIANO, Liber singularis regularum, 28,10: "Bonorum possessio datur parentibus et liberis intra annum, ex quo petere potuerunt, ceteris intra centum dies" (en KRUEGER, P., Collectio librorum iuris anteiustiniani (Berolini, apud Weimannos, 1878), II, p. 36 [hay traducción castellana de Ponssa de la Vega de Miguens, N., Reglas de Ulpiano (Buenos Aires, Lerner, 1970), p. 173]. En el mismo sentido, Inst. 3,9,8. 
d. C.), donde se nos informa que a quienes compete por razón de parentesco la bonorum possessio tienen cien días útiles para reclamarla ${ }^{26}$.

\section{ESQUEMA}

Podemos trazar un esquema de la petición de bonorum possessio. La similitud con la fórmula que transmite Teófilo y la utilización en un contexto latino, nos permite creer que en alguna medida tal esquema puede ser extrapolado al resto del Imperio Romano durante el siglo III. Helo aquí:

[Nombre del pretor (en dativo)] " $a b$ " [nombre de quien dirige la petición, con mención de su tutor o curador, si es el caso; se da su filiación y procedencia, como también otros datos para su identificación]; "rogo, domine, des mihi bonorum possessionem" [nombre de la persona de cuyos bienes se pide la posesión de los bienes, especificando parentesco]; "ex ea parte edicti qua" [tipo de heredero]; "bonorum possessionem dari te polliceris. Datum" [fecha].

La fórmula propiamente dicha, citada en estos documentos, es "des mihi bonorum possessionem", generalmente no abreviada. Es interesante notar la exactitud de las fuentes utilizadas por Teófilo, más de ciento cincuenta años después de caer en desuso la fórmula. Es probable, pues, la existencia de un formulario procesal en las bibliotecas jurídicas romanas, aún accesible en el siglo VI.

[Recibido el 2 de abril y aprobado el 12 de junio de 2009].

\section{BIBLIOGRAFÍA}

Adams, J. N., "Romanitas" and the Latin Language, en The Classical Quarterly 53.1 (2003).

Adams, J. N., Bilingualism and the Latin Language (Cambridge, Cambridge University Press, 2004),

Bancalari Molina, A., Relación entre la "Constitutio Antoniniana" y la "Imitatio Alexandri" de Caracalla, en Revista de Estudios Histórico-Jurídicos 22 (2000).

Baviera, J. - Furlani, J., Fontes Iuris Romani Anteiustiniani (Florentiae, apud S. A. G. Barbèra, 1940), II.

Bell, H. I., P. Giss. 40 and the "Constitutio Antoniniana", en Journal of Egyptian Archaeology 28 (1942).

Bell, H. I., The "Constitutio Antoniniana" and the Egyptian Poll-Tax, en Journal of Roman Studies 37 (1947) 1-2.

Codex Iustinianus (ed. Krüger).

Codex Theodosianus (ed. Mommsen).

D’Ors, A. - Gundel, H. G., Zur Constitutio Antoniniana (P. Giss. 40 I). Kurzberichte aus den Giessaner Papyrus-Sammlung Nro. 22 (Giessen, 1973).

D’Ors, A., Estudios sobre la “Constitutio Antoniniana”, en Emerita 11 (1943); Anuario

\footnotetext{
${ }^{26}$ CI. 6.9.2: "Impp. Severus et Antoninus AA. Crispino: Si bonorum possessio duntaxat tibi competit proximitatis nomine, habuisti spatium centum dierum utilium, ex quo eum defunctum scisti, ab bonorum possessionem amplectendam".
} 
de Historia del Derecho Español 15 (1944); Anuario de Historia del Derecho Español 17 (1946); Sefarad 6 (1946); Emerita 24 (1956).

D'Ors, A., Introducción al Estudio de los Documentos del Egipto Romano (Madrid, Instituto Antonio de Nebrija, 1948).

Eger, O., Agnitio bonorum possessionis vom Jahre 249 p. C., en Zeitschrift der SavignyStiftung rom. Abt. 32 (1911).

Gagos, T. - HeIlporn, P., A New "agnitio bonorum possessionis", en Gagos, T. - BAGNALL, R. S., Essays and Texts in Honor ofJ. David Thomas (Oakville, The American Society of Papyrologists, 2001).

GLÜCK, Chr. F., Ausführliche Erläuterungen der Pandecten (Erlangen, 1870), XXXVII.

Grenfell, B. P. - Hunt, A. S., The Oxyrrhynchus Papyri, Part IV (London, Egypt Exploration Society, 1904).

Heichelheim, F. M., The Text of the Constitutio Antoniniana and the Three Other Decrees of the Emperor Caracalla contained in Papyrus Gissensis 40, en Journal of Egyptian Archaeology 26 (1941).

Hunt, A. S., The Oxyrhynchus Papyri, Part IX (London, Egypt Exploration Fund, 1912); iv) P. Mich. 1946 (269 d.C.), en Gagos, T. - Heilporn, P., A new “agnitio bonorum possessionis", en Gagos, T. - Bagnall, R. S., Essays and Texts in Honor of J. David Thomas (Oakville, The American Society of Papyrologists, 2001).

Institutionum Graeca paraphrasis. Theophili Antecessori vulgo tributa, ad fidem librorum manu scriptorum recensuit, prolegominis, notis criticis, versione Latina instruxit $E$. $C$. Ferrini. Accedit espistula C. E. Zacharia von Ligenthal (reimpresión Aalen, Scientia, 1967), dos volúmenes.

Iustiniani Institutiones (ed. Krüger).

Janeras, S. (editor), Miscelània Papirògica Ramon Roca-Puig en el seu vuitantè aniversari (Barcelona, Fundació Salvador Vives Casajuana, 1987).

Jones, A. H. M., Another Interpretation of the "Constitutio Antoniniana", en Journal of Roman Studies 26 (1936) 2.

Katzoff, R., The Provincial Edict in Egypt, en Tijdschrift voor Rechtsgeschiedenis/ Revue d'Histoire du Droit 37 (1969) 3-4.

Keresztes, P., The "Constitutio Antoniniana" and the Persecutions of Caracalla, en The American Journal of Philology 91 (1970) 4.

KRELLER, H., Erbrechtliche Untersuchungen auf Grund der graeco-aegyptishcen Papyruskunden (Leipzig - Berlin, 1919).

LAISTNER, M. L. W., “Dediticii”: the Source of Isidore (Etym. 9.4.49-50), en Journal of Roman Studies 11 (1921).

Lenel, O., Das Edictum Perpetuum, ein Versuch zu seiner Wiederherstellung (3a edición, Leipzig, Bernhard Tauchnitz, 1927).

Liddell, H. G. - Scott, R., A Greek-English Lexicon (19a edición, Oxford, Clarendon Press, 1996).

MeYer, P. M., Griechische Papyri im Museum des oberhessischen Geschichtvereins zu Gissen (P. Giss.) (Leipzig - Berlin, Teubner, 1910).

MEYER, P. M., Juristische Papyri, Erklärung von Urkunden zur Einführung in die Juristische Papyruskunde (Berlin, Weidmannsche Buchhandlung, 1920).

MEYER, P. M., Juritische Papyri, Erklärung von Urkunden zur Einführung in die Juristische Papyruskunde, (Berlin, Weidmannsche Buchhandlung, 1920). 
Mitteis, L. - Wilcken, U., Grundzüge und Chrestomathie der Papyruskunde (Hildesheim, Georg Olms Verlagsbuchhandlung, 1963), II, 2.

Oliver, J. H., Free Men and "Dediticii, en The American Journal of Philology 73, (1955) 6.

Ortolan, J.-J.-E., Explicación histórica de las Instituciones del Emperador Justiniano (trad. cast., Madrid, 1884), II.

REA, J. R., The Oxyrhynchus Papyri, Part XLIII (London, Egypt Exploration Society, 1975).

Riccobono, S., Fontes Iuris Romani Anteiustiniani, Pars Prima: Leges (Florentiae, apud S. A. G. Barbèra, 1941),

Roberts, C. H. - Turner, E. G., Catalogue of the Greek and Latin Papyri in the John Rylands Library, Manchester (Manchester, 1952, IV.

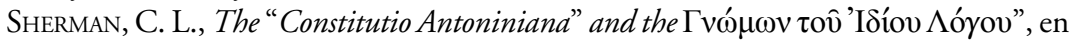
Transactions and Proceedings of the American Philological Association 59 (1928).

SHerwin-White, A. N., The Tabula of Banasa and the "Constitutio Antoniniana", en Journal of Roman Studies 63 (1973).

Thompson, E. M., An Introdution to Greek and Latin Paleography, (Oxford, Clarendon Press, 1912).

Wilhelm, A., Die "Constitutio Antoniniana”, en American Journal of Archaeology 38 (1934) 1.

Winter, J. G. y otros, Michigan Papyri, III (University of Michigan Studies, Humanistic Series 40, Ann Arbor, 1936). 